\title{
Colon Unfolding Via Skeletal Subspace Deformation
}

\author{
Sandra Sudarsky ${ }^{1}$, Bernhard Geiger ${ }^{1}$, Christophe Chefd'hotel $^{1}$, \\ and Lutz Guendel ${ }^{2}$ \\ ${ }^{1}$ Siemens Corporate Research Inc., Princeton, NJ 08540, USA \\ \{sandra.sudarsky, geiger. bernhard, christophe.chef dhotel\}@siemens.com \\ ${ }^{2}$ Siemens Medical Solutions, Forchheim, Germany
}

\begin{abstract}
We present an efficient method to digitally straighten a colon volume using mesh skinning, a technique well known in computer graphics to deform a polygonal mesh attached to a skeleton hierarchy. In our case, the colon centerline is used as the skeleton structure and the polyhedral model of the lumen as the skin that is to be deformed as the centerline is straightened. Once the colon has been straightened, we use standard rendering techniques to compute the virtual dissection. Our approach is significantly more efficient than previously proposed techniques.
\end{abstract}

\section{Introduction}

Virtual colonoscopy is a non-invasive screening procedure aimed at exploring the inner colonic surface in search for lesions. Standard methods in virtual colonoscopy simulate conventional colonoscopy by using virtual fly-throughs along the reconstructed colon. Major problems with this technique are the time required to navigate through the complex colon shape and the number of areas that are often left uninspected as they remain occluded behind Haustral folds. A number of techniques have been proposed to alleviate this problem, including Mercator projections [1, an unfolded cube display [2] and panoramic projections [3]. Recently, an alternative approach has emerged in the literature which proposes the use of virtual dissection of the colonic surface to speed up the inspection process. With this technique, the 3D model of the colon is cut open longitudinally and displayed as a single flat image. This approach has the potential of decreasing the inspection time and at the same time reducing the number of blind areas. However, it is well known that the colon lumen can not be flattened onto a plane without introducing some deformations 4. In this paper we describe an efficient virtual dissection technique based on mesh skinning [5] This is a well known technique used in computer animation to deform polygonal meshes such as the skin affixed to an articulated figure. We apply this technique to straighten the colon using the centerline as the skeleton and the polyhedral mesh that defines the colon lumen as the skin. 


\section{Previous Work}

A number of methods have been proposed to digitally straighten and unfold the colon to expose the entire colon lumen as a single image. Wang et al [7] propose a uniform sampling technique using planar cross sections orthogonal to the centerline. The results are satisfactory for portions of the colon that are fairly linear, but produce undesirable results in high curvature areas. This straightforward sampling can lead to single lesions being displayed more than once or missed completely. In [8], Wang et al propose a method to transform the colon into a straight cylinder-like shape based on the electrical field of a charged centerline. When the entire centerline is charged, the curved cross-sectional planes tend to diverge avoiding the double sampling problem. In practice however, the path is charged only locally and therefore there is no guarantee that the cross sections will not intersect. The method remains computationally very expensive.

Haker et al 9] propose a method to map the entire colon surface onto a flat surface using a conformal mapping, based on a discretization of the LaplaceBeltrami operator.

Bartroli et al 10] deal with the problems of double appearance of lesions and non-uniform sampling by casting rays that follow the negative gradient direction of a distance map generated from the centerline. These rays are curved and do not intersect. The distance between the ray origins and the hit surface point determines a height field. The height field is then unfolded and a non linear scaling is applied to compensate for distortions introduced by the non uniform sampling. The computational time for the entire process is in the range of hours.

Silver et al [11] propose an algorithm to unravel and deform a 3D volume. Their method is based on the computation of a volumetric skeleton using a reversible thinning procedure based on a distance transform. The skeleton can be interactively manipulated and the deformed volume reconstructed via an inverse transformation.

In the work of Zhang et al [12, the colon straightening is modeled as a solid elastic deformation process with special constraints and boundary conditions. The deformation model is described by a group of partial differential equations based on equilibrium and kinematic equations found in solid mechanics theory.

Hong et al [13] present an algorithm that flattens the colon in a conformal manner and minimizes global distortion. The conformal parameterization is solved using finite element methods to approximate a solution of an elliptic partial differential equation on surfaces. The entire process takes about 30 minutes for a 512 × 512 × 460 data set.

The approach of Sabry et al 14 is unique in that the colon is not straighten nor flatten. Instead, the colon surface is split into two halves along the centerline based on a mesh skinning technique. Each half is then assigned a virtual camera that performs a fly-over navigation.

We propose a novel approach to digitally dissect the colon which is significantly faster than all previously proposed techniques. We can generate a complete unfolding in under a minute in a standard PC. 


\section{Our Approach}

Our approach is based on a technique known as mesh skinning 57 often used in computer animation to deform a polygonal mesh attached to a skeleton hierarchy. In our case, the colon centerline is used as the skeleton structure and the polygonal model of the lumen as the skin that is to be deformed as the centerline is straightened. In this section we describe in detail the proposed algorithm as outlined in Figure 1. Given a CT data volume we compute a fully automated colon segmentation based on a threshold region growing operation. The centerline, a sequence of joints or points $\left\{c_{1}, \ldots, c_{n}\right\}$, is computed for all components in the threshold volume. Two consecutive centerline points define a bone or a link segment. As shown in Figure 2a, each centerline joint has an associated local coordinate system where the $z$ axis is oriented to line up with its corresponding bone and the other two axes are defined so that the angular rotation between consecutive frames is minimized. Finally, a polyhedral model of the colon surface is reconstructed using a marching cube algorithm. The resulting mesh consists of a set of triangles with vertices $\left\{v_{1}, \ldots, v_{p}\right\}$.

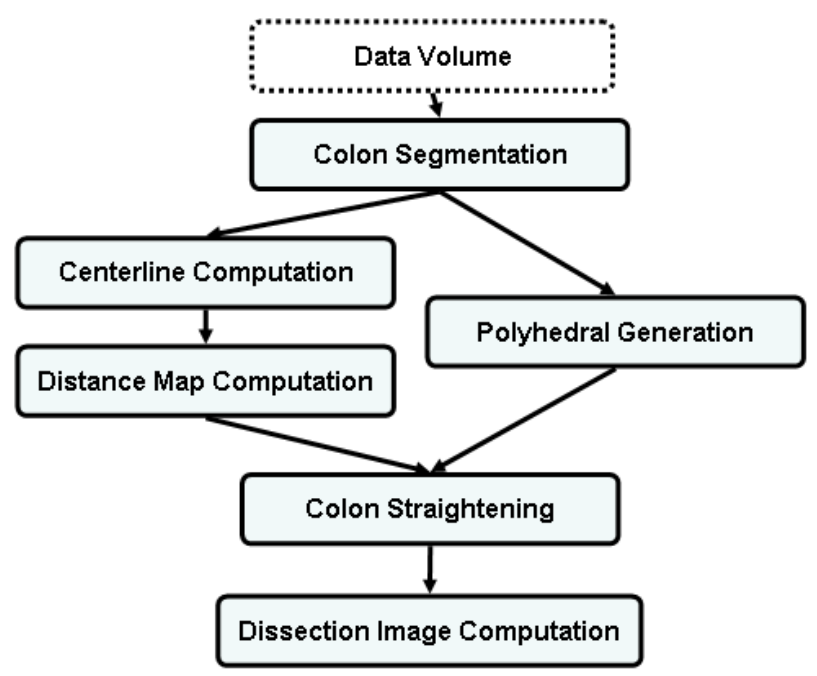

Fig. 1. The virtual dissection process

\subsection{Colon Straightening}

The straightening of the colon consists of three steps. The first step automatically assigns polyhedral vertices to bones and calculates weights $w_{i, j}$ that define how much influence a particular bone $j$ has on a vertex $v_{i}$ during the deformation process. To ensure a smooth skinning, each vertex in the mesh is associated with multiple joints. In order to define these weights, a distance map is computed which encodes for each voxel on the colon wall its nearest centerline point. The 


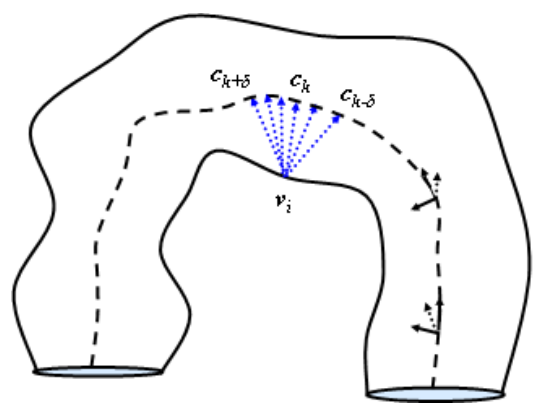

(a)

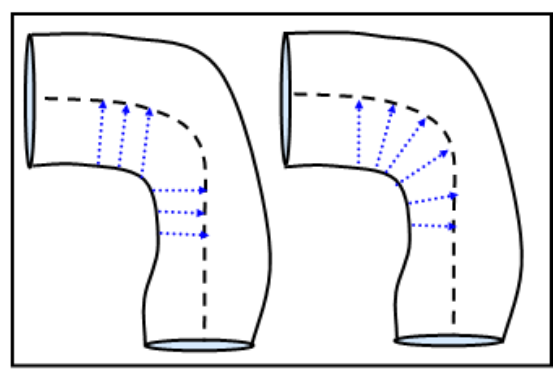

(b)

Fig. 2. (a) Centerline segments with corresponding coordinate systems. The nearest centerline point $c_{k}$ to vertex $v_{i}$ is found via a distance mapping. (b) Nearest centerline points before and after applying a smoothing step.

distance map is computed using a region growing technique based on the semiEuclidean distance transform [15] where the points that define the centerline are used as the seed points. Based on this map, each vertex $v_{i}$ of the polyhedral model is associated with its nearest point $c_{k}$ on the centerline (see figure 2a). Adjacent vertices on the polyhedron should have associated points on the centerline that are near each other. Because of the complicated structure of the colon, this is not always the case and could create severe distortion. To ensure a proper unfolding, we add a smoothing step (see figure $2 \mathrm{~b}$ ). For each vertex $v_{i}$ in the mesh, we average its nearest centerline point index $k$ with the indices of its adjacent vertices using several iterations.

We assign to each polyhedron vertex $v_{i}$ a neighborhood (of size $2^{*} \delta$ ) of centerline points symmetrically distributed around $c_{k}$ (see figure 2a). Weights $w_{i, j}$ are defined to be inversely proportional to the distances $d_{i, j}$ between the vertex $v_{i}$ and the centerline points $c_{j}$ in that neighborhood, namely

$$
w_{i, j}=\frac{1}{d_{i, j}} \times \frac{1}{T_{i}}
$$

where $T_{i}$ is the total sum of all those distances in the defined neighborhood

$$
T_{i}=\sum_{j=k-\delta}^{j=k+\delta} \frac{1}{d_{i, j}} .
$$

Note that to ensure that no undesired scaling will occur, this weight assignment satisfies equation 3 .

$$
\sum_{j=k-\delta}^{j=k+\delta} \frac{1}{w_{i, j}}=1 .
$$

In the second step, each centerline segment is aligned with respect to the previous segment to form a straight line $\left\{p_{1}, \ldots, p_{n}\right\}$ along the $z$ axis (Figure 3 ). 


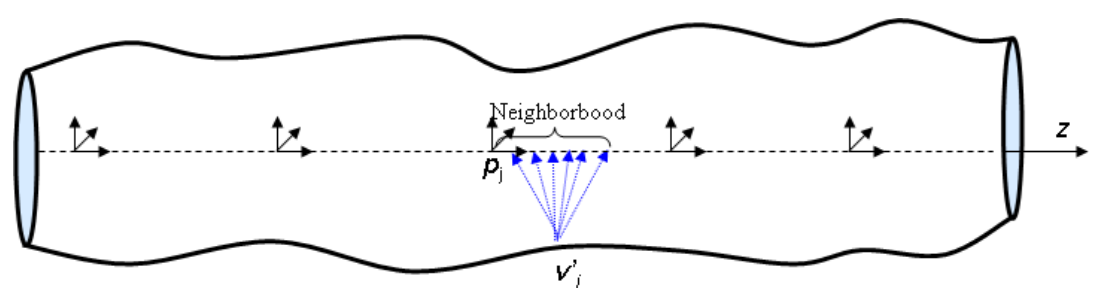

Fig. 3. Straighten colon

Starting at the origin, the $z$-coordinate of $p_{j}$ is given by $p_{j, z}=p_{j-1, z}+\left\|c_{j}-c_{j-1}\right\|$. A rotation matrix $M_{j}$ encodes the transformation of the world coordinate system into the local coordinate at $c_{j}$. Each matrix $M_{j}$ is computed by multiplying $M_{j-1}$ with a local rotation transforming frame $j-1$ to frame $j$.

The final step consists of recomputing the polyhedral vertex coordinates on the straighten polyhedron. The new value $v_{i}^{\prime}$ of $v_{i}$ is given by

$$
v_{i}^{\prime}=\sum_{j=k-\delta}^{j=k+\delta} w_{i, j}\left(p_{j}+M_{j}^{-1}\left(v_{i}-c_{j}\right)\right) .
$$

Note that since $M_{j}$ is a rotation matrix its transpose its used for $M_{j}^{-1}$.

\subsection{Dissection Computation}

The dissection is done in two passes. First, a low resolution sampling pass calculates the scaling parameters. At discrete points along the centerline, starting at the rectum and moving toward the cecum, few sample rays orthogonal to the central path are cast and the intersection of the rays with the polyhedron is calculated. For each ray the distance between the ray origin and the hit surface is computed. The average of these distances at a discrete centerline location is used to scale the dissection.

During the second pass, the unfolded image is generated. Rays are cast orthogonal to the central path and distributed uniformly covering more than $360^{\circ}$ (Figure 4). Using the material properties and the effect of the lights in the scene, the shading of the corresponding pixel is determined (Phong shading). Because more than $360^{\circ}$ are covered, the resulting image displays an overlap at the edges.

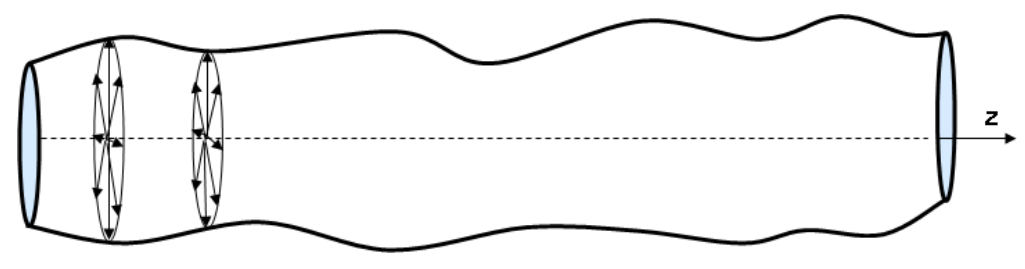

Fig. 4. Dissection computation 
To speed up the intersection calculation between the rays and the mesh, the triangles are sorted relative to the minimum z-component of their vertex coordinates. The dissection view computation can be displayed in a multiresolution setting. During the initial pass, a low resolution image is generated. Subsequent passes update the image to increase the resolution.

\section{Results}

Below we illustrate the results of the dissection computation. Figure 5 shows a colon polyhedral (with $285 \mathrm{k}$ triangles and $144 \mathrm{k}$ vertices) before and after the straightening procedure is applied. Figure 6 shows the corresponding virtual dissection. The image is split into four sections, starting at the rectum in the top left corner and ending up at the cecum in the bottom right. It illustrates the appearance of lesions in the dissection view and the corresponding findings in the endoluminal view. We provide a real time point-to-point correlation between the dissection and the corresponding 3D and 2D images. Lesions in the unfolded view tend to appear stretched out horizontally along the longitudinal axis, consistent with the conclusion of a recent study [4], while Haustral folds tend to appear perpendicular to the long axis of the colon.

The bottleneck of this technique is the dimension of the image used to display the flatten image. Table 1 lists average computation times for the major steps of the dissection algorithm for different image resolutions. The tests were performed on a $2.39 \mathrm{GHz}$ Pentium processor with $2 \mathrm{~GB}$ of RAM.

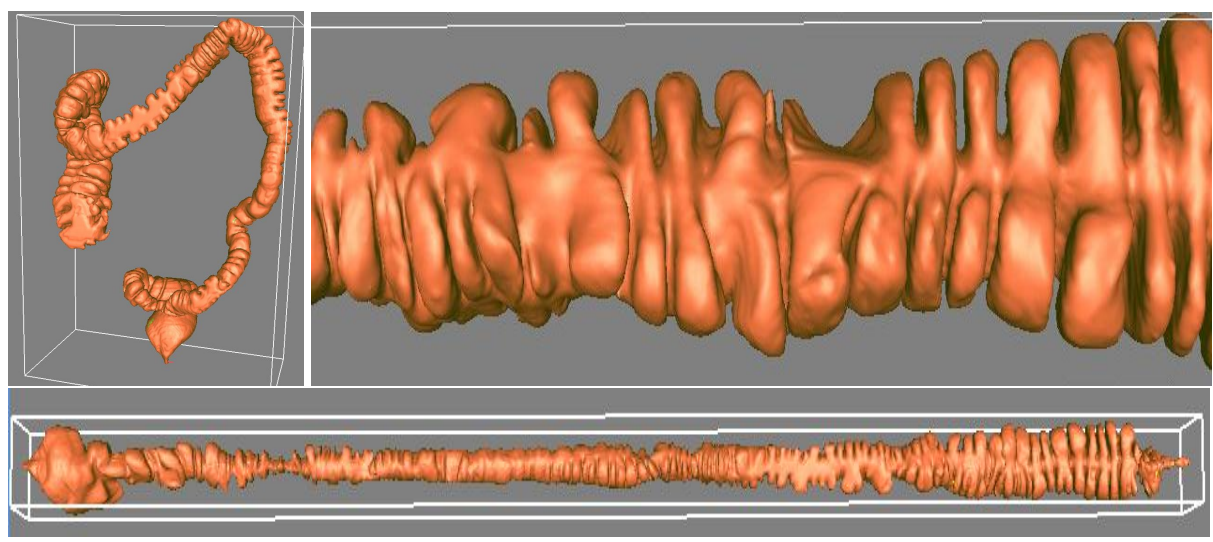

Fig. 5. Original colon volume and the results of straightening the centerline

Table 1. Dissection computation times for different image resolutions (in seconds)

\begin{tabular}{ccccc}
\hline $\begin{array}{c}\text { image } \\
\text { resolution }\end{array}$ & $\begin{array}{c}\text { Distance map } \\
\text { calculation }\end{array}$ & $\begin{array}{c}\text { colon } \\
\text { straightening }\end{array}$ & $\begin{array}{c}\text { diameters } \\
\text { calculation }\end{array}$ & $\begin{array}{c}\text { unfolding } \\
\text { rendering }\end{array}$ \\
\hline $4096^{*} 256$ & 1 & 1 & 8 & 28 \\
\hline $2048^{*} 128$ & 1 & 1 & 3 & 7 \\
\hline
\end{tabular}




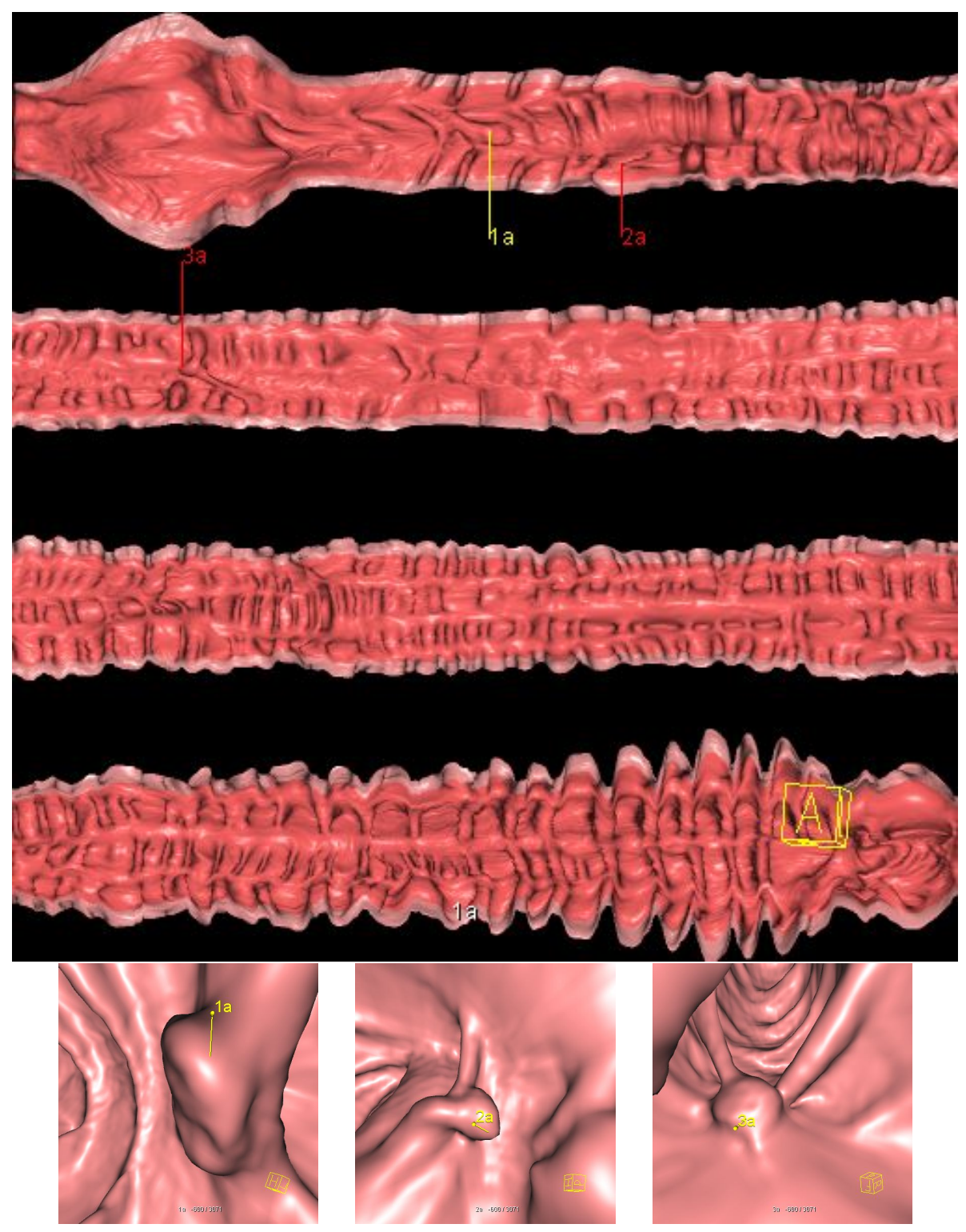

Fig. 6. Lesions in the dissection view and corresponding locations on the endoluminal view

\section{Conclusions}

We present a colon dissection algorithm using mesh skinning that is significantly faster than previously published techniques. The method has been tested on 30 datasets with good results. Problems arise in cases that have incomplete 
distension and cannot be aligned automatically on a consistent centerline. Clinical evaluation is planned to fully evaluate the diagnostic value of the proposed technique. Since deformations are liable to occur, it is critical that radiologists become familiar with the appearance of normal and abnormal colon features in the virtual dissection. Improvements of the method for cases where the colon is not well distended are subject of future research.

\section{References}

1. Paik, D., Beaulieu, C., Jeffrey, R., C.A., Karadi, C., Napel, S., Visualization modes for CT colonography using Cylindrical and Planar Map Projections, J. Comput Assist. Tomogr.,vol. 24(2), 179-188 (2000).

2. Vos, F., Serlie, I., van Gelder, R., Post, F., Truyen, R., Gerritsen, F., Stoker, J., Vossepoel, A.: A New Visualization Method for Virtual Colonoscopy. In: Niessen, W.J., Viergever, M.A. (eds.) MICCAI 2001. LNCS, vol. 2208, pp. 645-654. Springer, Heidelberg (2001)

3. Geiger, B., Chefd'hotel, C., Sudarsky, S.: Panoramic Views for Virtual Endoscopy. In: Duncan, J.S., Gerig, G. (eds.) MICCAI 2005. LNCS, vol. 3749, pp. 662-669. Springer, Heidelberg (2005)

4. Johnson K., Johnson C., Fletcher J., MacCarty R., Summers R. CT colonography using 360-degree virtual dissection: a feasibility study. AJR Am J Roentgenol, 186:9095, (2006).

5. Lewis, J., Cordner, M., Fong, N., Pose Space Deformations: A Unified Approach to Shape Interpolation and Skeleton-Driven Deformation, proceedings of ACM SIGGRAPH, 165-172, (2000).

6. Yang, X., Zhang, J., Realistic Skeleton Driven Skin Deformation, Lecture Notes in Computer Science (TSCG 2005), Springer-Verlag,(2005).

7. Wang, G., Vannier, M., Unraveling the GI tract by spiral CT, SPIE, 307-315, (1995).

8. Wang, G., McFarland, E., Brown, B., Vannier, M., GI tract unraveling with curved cross sections. IEEE Transactions on Medical Imaging, vol. 17, no. 2, (1998).

9. Haker, S., Angenent, S., Tannenbaum, A., Kikinis, R., Nondistorting Flattening for Virtual Colonoscopy, MICCAI, 358-366, Springer-Verlarg, (2000).

10. Vilanova Bartrolí, A., Wegenkittl, R., König, A., Gröller, E., NonLinear Virtual Colon Unfolding, Proc. IEEE Visualization, 411:420, (2001).

11. Silver, D., Gagvani, N., Unwinding the Colon, Medicine Meets Virtual Reality (MMVR), (2002).

12. Zhang, Z., Ackerman M., Li, J., Colon straightening based on an elastic mechanics model, ISBI, IEEE, 292-295, (2004).

13. Hong, W., Gu, X., Qiu, F., Jin, M., Kaufman, A, Conformal Virtual Colon Flattening, SPM, Cardiff Wales, 85-93, (2006).

14. Sabry Hassouna, M., Farag, A., and Falk, R., "Virtual Fly-Over: A New Visualization Technique For Virtual Colonoscopy," MICCAI, 381-388, (2006).

15. Gibson, S., Calculating the Distance Map for Binary Sampled Data, Technical Report TR99-26, Mitsubishi, (1999). 